\title{
Percepção dos graduandos de Odontologia quanto à atividade prática de estimativa de idade realizada em uma disciplina de Odontologia Legal
}

Johnys Berton Medeiros da Nóbrega*; Raphael Cavalcante Costa*; Laíse Nascimento Correia Lima**, Patrícia Moreira Rabello***; Ana Maria Gondim Valença***; Bianca Marques Santiago***

\begin{abstract}
RESUMO
O estudo objetiva identificar as facilidades e dificuldades vivenciadas pelos alunos em atividade prática de estimativa de idade proposta pela disciplina de Odontologia Legal, verificando sua percepção quanto à funcionalidade e/ou importância da atividade na sua formação acadêmica. Trata-se de uma pesquisa de campo aplicada, quantitativa, exploratória, documental e analítica, do tipo transversal. Durante uma atividade prática da disciplina de Odontologia Legal da Universidade Federal da Paraíba, aplicou-se um questionário com perguntas abertas e fechadas acerca das facilidades e dificuldades vivenciadas na aplicação de um método de estimativa de idade e da percepção dos alunos quanto a funcionalidade e importância da atividade. A amostra $(n=52)$ foi composta pelos alunos matriculados na disciplina de Odontologia Legal nos períodos letivos 2014/2 e 2015/1, que participaram da atividade prática. Os dados foram analisados descritivamente. A opção "Excelente" foi maioria em três dos quatro critérios avaliados.
\end{abstract}

Estudante do Curso de Odontologia, UFPB.

Professora do Departamento de Odontologia, UFMA.

Professora do Departamento de Clínica e Odontologia Social, UFPB.

Como pontos positivos, destacaram-se a aplicabilidade $(19,0 \%)$, fixação do conteúdo $(17,2 \%)$ relevância para formação profissional $(12,1 \%)$ e praticabilidade $(12,1 \%)$. Como pontos negativos e dificuldades encontradas foram ressaltadas a subjetividade do método em estudo (25,0\%), a má qualidade das projeções radiográficas $(18,2 \%)$, a restrição de aplicação do método a crianças e adolescentes $(9,1 \%)$ e a necessidade de estudo prévio $(9,1 \%)$. Em conclusão, a atividade prática de estimativa de idade contribuiu para a formação profissional dos alunos, sendo a aplicabilidade do método o ponto positivo mais frequentemente apontado, enquanto a subjetividade do mesmo se constituiu na maior dificuldade vivenciada pelos graduandos.

Descritores: Determinação de Idade pelos Dentes. Odontologia Forense. Educação Superior.

\section{INTRODUÇÃO}

Com a constante mudança nas sociedades e a intensa densidade de aconte- 
cimentos cotidianos, faz-se necessária uma discussão contínua sobre a adequação curricular das escolas de ensino superior. Todavia, é preciso ter consciência que mudanças curriculares levam certo tempo para que os resultados almejados apareçam e sejam suficientes para transformar o ambiente formador em que se encontram, através da adaptação dos seus integrantes ${ }^{1}$.

Pinheiro (2009) $)^{2}$ mapeou, na literatura nacional, estudos que tratavam da formação odontológica no Brasil entre 1992 e 2005, e observou que grande parte desses estudos conclui sobre a necessidade de mudanças na formação do cirurgião-dentista, com destaque para a necessidade de alterações curriculares e o ensino voltado para as necessidades sociais, bem como a integração ensino-serviço.

Cada vez mais o ensino forense e a formação em odontologia vêm sendo discutido e refletido, principalmente por meio das reuniões da Associação Brasileira de Ensino Odontológico (ABENO). Especialmente em 2012, ocorreu juntamente com a $47^{\mathrm{a}}$ reunião da $\mathrm{ABENO}$, uma reunião paralela de professores de Odontologia Legal. Essa entidade, juntamente com a Associação Brasileira de Ética e Odontologia Legal (ABOL), recomenda o ensino da Odontologia Legal com uma disciplina dividida em pelo menos dois períodos de 60 horas cada, com abordagem da Deontologia e Diceologia na primeira parte e Odontologia Legal Aplicada na segunda ${ }^{3}$.

No tocante ao ensino da Odontologia Legal, é interessante ressaltar o empenho de profissionais e docentes da área para a aproximação da teoria com a prática forense como forma de dinamização das disciplinas, tentando potencializar o aprendizado.
Uma das questões frequentes na prática da Odontologia Legal é a realização da estimativa da idade, quando é possível utilizar tanto a análise clínica dental como a interpretação de radiografias dos elementos dentários, sendo a última uma técnica mais confiável $^{4}$. No Brasil, especificamente para as ciências forenses, a tabela de mineralização dos dentes permanentes de Nicodemo, Moraes e Médici Filho $(1974)^{5}$ tem sido utilizada e recomendada por ser a única a ter uma população brasileira como referência. $\mathrm{O}$ método requer a utilização de radiografia panorâmica para a averiguação acerca do estágio de mineralização dentária, e então buscar, na tabela de referência do mesmo, a provável faixa de idade do indivíduo ${ }^{5}$.

Com base no exposto, o presente trabalho se propôs a identificar as facilidades encontradas e dificuldades vivenciadas pelos alunos em atividade prática de estimativa de idade proposta pela disciplina de Odontologia Legal, verificando a percepção dos alunos quanto à funcionalidade e/ou importância da atividade na sua formação acadêmica.

\section{METODOLOGIA}

O estudo consistiu em uma pesquisa de campo aplicada, sendo quantitativo, exploratório, documental e analítico, do tipo transversal $^{6}$, e foi realizado na Universidade Federal da Paraíba (UFPB), nas dependências do curso de graduação em Odontologia, vinculado ao Centro de Ciências da Saúde, durante as aulas práticas do componente curricular de Odontologia Legal.

Esta pesquisa obedeceu aos aspectos éticos prescritos na Resolução no 466/12, do Conselho Nacional de Saúde (CNS), e foi aprovada pelo Comitê de Ética em Pesquisa 
(CEP) do Centro de Ciências da Saúde (CCS) da UFPB, (CAAE 25898113.8.0000.5188).

Durante o componente curricular de Odontologia Legal da UFPB, realiza-se uma atividade prática, na qual três radiografias panorâmicas, de indivíduos cujas idades são conhecidas somente pelos docentes, são projetadas e os alunos orientados a interpretálas, utilizando a tabela de mineralização dos dentes permanentes proposta por Nicodemo, Moraes e Médici Filho (1974) ${ }^{5}$, com o intuito de estimar a idade dos indivíduos.

O universo do estudo compreendeu todos os alunos regularmente matriculados na disciplina de Odontologia Legal em dois períodos consecutivos (2014/2 e 2015/1). A amostra $(n=52)$ foi composta pelos que compareceram à aula prática de estimativa de idade e, na mesma, preencheram as fichas fornecidas para interpretação radiográfica e ainda concordaram em cedê-la para o estudo mediante a assinatura do Termo de Consentimento Livre e Esclarecido.

A equipe envolvida no estudo aplicou um questionário, testado previamente em estudo piloto ${ }^{7}$, com perguntas abertas e fechadas acerca das facilidades e dificuldades vivenciadas na aplicação do método e da percepção dos alunos quanto a sua funcionalidade e importância para a formação acadêmica.

Os dados foram tabulados e analisados descritivamente no programa estatístico SPSS ${ }^{\circledR}$ (IBM Analytics, Armonk, NY, EUA), versão 20.0 .

\section{RESULTADOS}

A tabela 1 mostra a disposição dos resultados totais das duas turmas. A opção "Excelente" foi maioria em três dos quatro critérios avaliados. $\mathrm{Na}$ variável em que
"Excelente" (30,8\%) não liderou as opiniões, ficou como a segunda mais citada, em seguida de "Bom" (51,9\%).

Na tabela 2 são visualizados os pontos positivos. Ao todo, foram 15 pontos diferentes citados dentre 58 coletados, uma vez que cada aluno poderia expressar mais de um ponto positivo ou simplesmente não apontar nenhum. O mais presente foi a aplicabilidade $(19,0 \%)$, seguido de fixação do conteúdo $(17,2 \%)$, relevância para formação profissional $(12,1 \%)$ e praticabilidade $(12,1 \%)$, com destaque para o expressivo número de alunos que não citaram nenhum ponto $(19,2 \%$; $\mathrm{n}=10$ ).

Os resultados das questões abertas referentes aos pontos negativos estão representados na tabela 3; subjetividade do método $(25,0 \%)$ e projeção de má qualidade $(18,2 \%)$ foram os mais apontados. Dezenove alunos $(36,5 \%)$ não opinaram nesta questão.

\section{DISCUSSÃO}

Na UFPB, a disciplina de Odontologia Legal possui carga horária de 60 horas e está inserida no último ano da graduação, no $9^{\circ}$ período, juntamente com uma elevada carga horária clínica, além de ser o período em que o aluno apresenta seu Trabalho de Conclusão de Curso, e isso pode gerar desinteresse do aluno pela disciplina, consequentemente, pela área de trabalho.

A última reestruturação da grade curricular em Odontologia da UFPB aconteceu em 2002, segundo a Resolução $\mathrm{N}^{\circ}$ 37/2002 aprovada pelo Conselho Superior de Ensino, Pesquisa e Extensão (CONSEPE) da $\mathrm{UFPB}^{8}$, e a disciplina de Odontologia Legal se encontra conforme os parâmetros de carga horária, divisão no fluxograma e conteúdos abordados, recomendados pela ABOL e a 
$\mathrm{ABENO}^{3}$, porém, ainda não está em completo acordo pelo posicionamento das disciplinas no fluxograma.

A primeira parte do ensino, que está inserida na disciplina de Ética e Legislação
Odontológica, não é ministrada no início das práticas clínicas, mas sim, no oitavo período, um período antes da disciplina de Odontologia Legal, onde são ministrados os conteúdos da segunda parte.

Tabela 1. Frequências absoluta e percentual do nível de aceitabilidade para os critérios avaliados.

\begin{tabular}{c|cccccccc}
\hline Critérios & \multicolumn{2}{c}{$\begin{array}{c}\text { Alcance dos } \\
\text { objetivos } \\
\text { propostos }\end{array}$} & \multicolumn{2}{c}{$\begin{array}{c}\text { Adequação da } \\
\text { atividade ao } \\
\text { conteúdo }\end{array}$} & \multicolumn{2}{c}{$\begin{array}{c}\text { Tempo } \\
\text { disponível para } \\
\text { preparação da } \\
\text { atividade }\end{array}$} & \multicolumn{2}{c}{$\begin{array}{c}\text { Contribuição da } \\
\text { atividade para a } \\
\text { formação }\end{array}$} \\
& $\mathbf{n}$ & $\mathbf{\%}$ & $\mathbf{n}$ & $\mathbf{\%}$ & $\mathbf{n}$ & $\mathbf{\%}$ & $\mathbf{n}$ & \% \\
\hline Insuficiente & 01 & 1,9 & 00 & 0,0 & 01 & 1,9 & 01 & 1,9 \\
Regular & 08 & 15,4 & 03 & 5,8 & 03 & 5,8 & 02 & 3,9 \\
Bom & 27 & 51,9 & 12 & 23,1 & 16 & 30,8 & 18 & 34,6 \\
Excelente & 16 & 30,8 & 37 & 71,1 & 32 & 61,5 & 31 & 59,6 \\
\hline Total & 52 & 100,0 & 52 & 100,0 & 52 & 100,0 & 52 & 100,0 \\
\hline
\end{tabular}

Tabela 2 - Frequências absoluta e percentual dos pontos positivos apontados nos questionários.

\begin{tabular}{l|cc}
\hline \multicolumn{1}{c|}{ Pontos Positivos } & n & \% \\
\hline Aplicabilidade & 11 & 19,0 \\
Fixação do conteúdo & 10 & 17,2 \\
Relevância para a formação profissional & 7 & 12,1 \\
Praticabilidade & 7 & 12,1 \\
Dinamicidade & 5 & 8,6 \\
Aproximação com a idade real & 4 & 6,9 \\
Opção para estimativa de idade & 3 & 5,2 \\
Relevância para a carreira em Odontologia Legal & 3 & 5,2 \\
Método interessante & 2 & 3,5 \\
Necessidade de conhecimento prévio & 1 & 1,7 \\
Utilização de radiografias panorâmicas & 1 & 1,7 \\
Interatividade & 1 & 1,7 \\
Contribuição dos conhecimentos radiológicos & 1 & 1,7 \\
Método brasileiro & 1 & 1,7 \\
Baixo custo & 1 & 1,7 \\
\hline Total & 58 & 100,0 \\
\hline
\end{tabular}


Tabela 3. Frequências absoluta e percentual dos pontos negativos apontados nos questionários.

\begin{tabular}{l|cc}
\hline \multicolumn{1}{c|}{ Pontos Negativos } & n & \% \\
\hline Subjetividade do método & 11 & 25,0 \\
Projeção de má qualidade & 8 & 18,3 \\
Método aplicado apenas para crianças e adolescentes & 4 & 9,1 \\
Necessidade de estudo prévio & 4 & 9,1 \\
Alta amplitude de idade estimada & 3 & 6,8 \\
Eficácia indesejada na concordância das idades & 3 & 6,8 \\
Tempo insuficiente & 2 & 4,5 \\
Dificuldade do método & 2 & 4,5 \\
Necessidade de radiografia panorâmica & 2 & 4,5 \\
Poucas práticas & 2 & 4,5 \\
Condições impróprias & 1 & 2,3 \\
Necessita boa percepção radiográfica & 1 & 2,3 \\
Poucos exemplos & 1 & 2,3 \\
\hline Total & 44 & 100,0 \\
\hline
\end{tabular}

A disciplina de Odontologia Legal na graduação é motivo de reflexão devido à intensa evolução da sociedade. É notória a necessidade de adequação dos cursos de graduação nesse contexto para atender às demandas que surgem, sendo este aspecto discutido em vários simpósios e congressos da área no Brasil ${ }^{9}$.

Da década de 1970 para os dias atuais, a Odontologia Legal, através da estimativa de idade, passou a ser estudada por mais autores $^{10-14}$ e vem progredindo em vários horizontes, exemplos disso são a criação da ABOL em 1996, e mais recentemente em 2014 da Revista Brasileira de Odontologia Legal (RBOL). Esse desenvolvimento veio após alguns episódios em que a utilização da Odontologia Legal foi noticiada internacionalmente ${ }^{15,16}$, e em todos, a radiologia esteve presente dando o suporte necessário com a comparação de tomadas radiográficas do corpo com uma do mesmo, quando vivo $^{17}$.

Apesar de 59,6\% dos alunos assinalarem "Excelente" e 34,6\% opinarem
"Bom" para "Contribuição da atividade para a formação" e na parte de questões abertas $12,1 \%$ referirem que um ponto positivo foi a relevância da atividade para a formação profissional, apenas 5,2\% explicitaram que a atividade teve relevância para a carreira em Odontologia Legal. Esse pequeno número interessado em seguir carreira em Odontologia Legal pode estar relacionado a vários fatores, inclusive à configuração do atual fluxograma de disciplinas do curso, oferecendo o referido componente curricular somente no nono período. Outro fator que pode influenciar tal processo de descontinuidade da graduação com o seguimento na área, é o expressivo número de programas de pós-graduação e cursos de aperfeiçoamento e especialização das outras áreas frente à Odontologia Legal.

Segundo o perfil e tendências do Cirurgião-Dentista brasileiro publicado em $2010^{18}$, a Odontologia Legal estava entre as especialidades menos numerosas do país dentre as 19 legalmente reconhecidas pelo CFO naquela época, e em 2007, só havia um 
programa de pós-graduação com área de concentração na Odontologia Legal em todo Brasil, e isso naturalmente afeta o mercado de trabalho.

Em contrapartida aos pontos negativos apontados relativos à radiologia, os positivos citados foram "Utilização de radiografias panorâmicas" e "Contribuição dos conhecimentos radiológicos". A utilização de radiografia panorâmica para aplicação do método é vista como um ponto negativo por alguns talvez pela pouca quantidade de institutos médico-legais e laboratórios forense equipados com aparelhagem de radiologia, e dificuldade de manejo no posicionamento para a execução da radiografia post-mortem devido à rigidez cadavérica, onde o profissional não pode contar com a ajuda do indivíduo. Esse fato sugere que seja desenvolvido um estudo de levantamento da prevalência de aparelhos radiológicos em tais órgãos.

"Método aplicado apenas para crianças e adolescentes" aparece em número relativamente expressivo comparado a outros pontos negativos, o que pode representar a necessidade de atividades práticas com outros métodos de estimativa de idade que tenham maior abrangência de idade, assim contribuindo ainda mais para a formação profissional dos alunos, um dos pontos positivos mais elencados. Um exemplo seria o método de Camargo (1994) ${ }^{10}$, que abrange indivíduos de 15 a 65 anos, não se baseia na mineralização dentária, mas também é aplicado por meio de radiografias.

A subjetividade do método apontada nos pontos negativos, associada à má qualidade da projeção das imagens radiográficas, pode ser alusiva ao fato de o método necessitar de treinamento para identificar com segurança em que estágio de mineralização cada dente está. Por ser o primeiro contato com a execução prática de um método de estimativa de idade e não possuírem segurança na escolha do estágio, os alunos confundiram isso com uma possível subjetividade que o método possa ter. Alguns, conscientes da necessidade de tal treinamento para determinação do estágio de mineralização com mais garantia, ainda reportaram como ponto negativo "Necessidade de estudo prévio".

Além de má qualidade da projeção radiográfica, também foram apontados "Necessidade de radiografia panorâmica" e "Necessidade de boa percepção radiográfica". Mas, na área Forense, e mais especificamente na Odontologia Legal, a radiologia contribui possibilitando vários métodos de estimativa de idade através das radiografias panorâmica, de crânio e face.

No estudo piloto de Corlett et al. $(2015)^{7}$, os resultados foram compatíveis com os da presente pesquisa. Nas questões fechadas, os resultados se apresentaram, em sua maioria, com a mesma distribuição, e na parte subjetiva, os pontos positivos e negativos mais citados, respectivamente, foram "Fixação do conteúdo" $(16,6 \%, n=9)$ e "Subjetividade do método" $(28,6 \%, \mathrm{n}=10)$. A ausência de dados ocorreu em 17,1\% ( $n=6)$ nos pontos negativos e em 3,4\% (n=2) nos positivos, se mostrando bem inferior ao que aconteceu neste estudo.

Uma das possíveis causas para a ausência de respostas nos pontos positivos das questões abertas poderia ser o fato de o questionário ter sido aplicado após a aula expositiva e execução da atividade prática acerca do método de estimativa de idade, gerando certa fadiga ao aluno. Com isso, nas 
questões objetivas, que se apresentaram antes no questionário, a maioria dos alunos assinalou a opção "Excelente" para "Adequação da atividade ao conteúdo" e "Contribuição da atividade para a formação", evitando expressar as duas variáveis como ponto positivo na avaliação aberta, situada na parte final do questionário, uma vez que "Fixação do conteúdo" e "Relevância para a formação profissional" figuraram entre os três mais citados.

Da mesma forma, uma possível explicação para a ausência de respostas nos pontos negativos seria o fato de, na parte fechada, em três dos quatro quesitos, a opção "Excelente" ter sido registrada. Também se nota certa polarização dos pontos negativos citados na subjetividade do método e na má qualidade da projeção.

O estudante de odontologia é submetido a um processo de ensinoaprendizagem, no qual ele é capacitado para desempenhar sua função profissional, agindo dentro de um padrão de respeito ético baseado em princípios humanos e técnico-científicos, porém, esse processo nem sempre acompanha a velocidade de transformação que o perfil do graduando apresenta, e se torna ineficiente quando não encontra uma boa articulação metodológica dinâmica e norteadora das necessidades para a formação ${ }^{19}$.

Quando a grade curricular está alicerçada na doença e não no paciente, é necessário recorrer ao que é sinalizado como necessidade pela epidemiologia, economia e sociedade, para que se tenha uma formação completa9. As Diretrizes Curriculares Nacionais $(\mathrm{DCN})^{20}$ para os cursos de Odontologia recomendam que além da capacitação nas Ciências Odontológicas, Biológicas e da Saúde, também seja realizada nas Ciências Humanas e Sociais, e existe uma tendência maior do graduando valorizar mais as atividades curativistas que as discussões éticas devido ao perfil de formação atual ${ }^{21,22}$.

Frequentemente alunos ingressam na graduação de odontologia com uma especialidade predefinida e direcionam sua formação em outro rumo devido a determinadas prioridades tomadas desde cedo, e isso pode transformar o ensino forense na odontologia em um desafio. Lino-Júnior et al. $(2015)^{9}$ concluíram que é preciso refletir e discutir para evoluir, e os principais pontos dessa discussão são a nova geração de profissionais; o trabalho em equipe; a apresentação de diferentes possibilidades de mercado para o estudante; e os desafios futuros para o fortalecimento da Odontologia Legal como uma importante e necessária vertente da área da saúde.

\section{CONCLUSÕES}

Os alunos avaliaram, em sua maioria, como excelente ou boa a contribuição proporcionada pela atividade prática de estimativa de idade para a sua formação acadêmica, e alguns também explicitaram isso em forma de pontos positivos, confirmando essa contribuição. As maiores dificuldades encontradas foram a subjetividade na aplicação da tabela de mineralização dos dentes permanentes de Nicodemo, Moraes e Médici Filho e a má qualidade das projeções radiográficas da atividade.

\section{ABSTRACT \\ Dental undergraduate students' perceptions regarding the practical activity of age estimation made in a course of Forensic Dentistry}

This study aimed to identify the facilities and difficulties experienced by students in a 
practical activity of age estimation proposed by the Forensic Dentistry curse, checking their perception regarding functionality and or importance of the activity in their academic training. This is a cross-sectional study with a quantitative and analytical approach. During a practical activity of the Forensic Dentistry curse at Federal University of Paraíba, a questionnaire was applied with open and closed questions about the facilities and difficulties experienced in the application of an age estimation method. Besides, the perception of students regarding the functionality and importance of the activity was asked. The sample $(n=52)$ was composed of students enrolled in the Forensic Dentistry discipline in two consecutive academic periods (2014/2 and 2015/1), which participated in the practical activity. Data were descriptively analyzed. The option "Excellent" was the most cited in three of the four criteria evaluated. As positive points, applicability (19.0\%), content (17.2\%), relevance for professional training $(12.1 \%)$ and practicability $(12.1 \%)$ stood out. The negative points and difficulties found were the method's subjectivity (25.0\%), poor quality of radiographic projections $(18.2 \%)$, restricted applicability to children and adolescents $(9.1 \%)$ and need for a previous study $(9.1 \%)$. In conclusion, the practical activity of age estimation contributed to the students' training, and the method applicability was the positive point most often cited, while the method's subjectivity was the main difficulty experienced by undergraduate students.

Descriptors: Age Determination by Teeth. Forensic Dentistry. Education, Higher.

\section{REFERÊNCIAS}

1. Toassi RFC, Stobäus CD, Mosquera JJM, Moysés SJ. Currículo integrado no ensino de Odontologia: novos sentidos para a formação na área da saúde. Interface. 2012; 16(41):529-42. [Acesso em: 27/06/2016]. Disponível em: http://www. scielo.br/scielo.php?script=sci_arttext\&pi $\mathrm{d}=$ S1414-3283201 2000200018

2. Pinheiro FMC, Nóbrega-Therrien $M$, Almeida MEL, Almeida MI. A formação do cirurgião-dentista no Brasil: contribuições de estudos para a prática da profissão. RGO. 2009; 57(1):99-106. [Acesso em: 27/06/2016]. Disponível em: http://www.revistargo.com.br/ojs/index.p hp/revista/article/viewArticle/648

3. Associação Brasileira de Ensino Odontológico. ABOL orienta sobre conteúdos da disciplina de Odontologia Legal. [Acesso em: 27/06/2016]. Disponível em: http://www.abeno.org. br/noticias/noticia020.php

4. Oliveira OF, Fernandes MM, Daruge Júnior E, Melani RFH, Paranhos LR. Estimativa da idade por meio de radiografias panorâmicas. RGO. 2010; 58(2):203-6. [Acesso em: 27/06/2016]. Disponível em: http://revodonto. bvsalud.org/pdf/rgo/v58n2/a10v58n2.pdf

5. Nicodemo RA, Moraes LC, MédiciFilho E. Chronological Table of Mineralization of Permanent Teeth Among Brazilians. Rev Fac Odontol São José Campos. 1974; 3(1):55-56.

6. Lakatos EM, Marconi MA. Metodologia Científica. 5 ed. São Paulo: Atlas, 2007.

7. Corlett IA, Lins RBE, dos Santos EJL, Santiago BM. Graduandos em odontologia e seu primeiro contato com a estimativa de idade dental: um estudo piloto. RBOL. 2015; 2(1):1-8. [Acesso em: 27/06/2016]. Disponível em: http://portalabol.com.br/rbol/index.php/R BOL/article/view/16

8. Conselho Superior de Ensino, Pesquisa e Extensão. Aprova o Projeto PolíticoPedagógico do Curso de Graduação em 
Odontologia, do Centro de Ciências da Saúde, Campus I, desta Universidade, revoga a Resolução UFPB/CONSEPE $n^{\circ}$ $71 / 83$ e dá outras providências. Resolução n. 37 , de 13 e 14 de junho de 2002, João Pessoa.

9. Lino-júnior HL, Gabriel M, DarugeJúnior E, Silva RHA. Ensino de Odontologia Legal no Brasil: um convite à reflexão. Rev ABENO. 2015; 15(2):3846. [Acesso em: 27/06/2016]. Disponível em: http://revabeno.emnuvens.com.br/ revabeno/article/view/161

10. Camargo JR. Estimativa da idade, após os 15 anos, utilizando-se das medidas da câmara pulpar e do canal radicular de dentes humanos, através de radiografias padronizadas. 1994. 62 f. Dissertação (Mestrado em Ciências) - Faculdade de Odontologia de Piracicaba, Universidade Estadual de Campinas, Piracicaba. 1994. [Acesso em: 27/06/2016]. http://www.bi bliotecadigital.unicamp.br/document/?co $\mathrm{de}=$ vtls000080062 \& print $=\mathrm{y}$

11. Saliba CA, Daruge E, Gonçalves R, Saliba TA. Estimativa da idade pela mineralização dos dentes, através de radiografias panorâmicas. ROBRAC. 1997; 6(22):14-16. [Acesso em: 27/06/2016]. Disponível em: http://seer. ufmg.br/index.php/arquivosemodontologi a/article/view/1601

12. Cornélio-Neto WL. Estimativa da idade pela mineralização dentária dos terceiros molares através de radiografias panorâmicas. 2000. 162 f. Dissertação (Mestre em Biologia Buco-Dental) Faculdade de Odontologia de Piracicaba, Universidade Estadual de Campinas, Piracicaba, 2000. [Acesso em: 27/06/2016]. Disponível em: http://www. bibliotecadigital.unicamp.br/document/?c ode $=$ vtls000205991

13. Costa FE. Estimativa de idade em radiografias panorâmicas através dos estágios de calcificação de Nolla. 2001. 80 f. Dissertação (Mestre em Biologia BucoDental) - Faculdade de Odontologia de Piracicaba, Universidade Estadual de Campinas, Piracicaba, 2001. [Acesso em: 27/06/2016]. Disponível em: http://www. bibliotecadigital.unicamp.br/document/?c ode $=$ vtls000226235

14. Almeida CSL. Estimativa de idade por radiografias panorâmicas em indivíduos melanodermas. 2002. 171 f. Tese (Doutorado em Biologia Buco-Dental) Faculdade de Odontologia de Piracicaba, Universidade Estadual de Campinas, Piracicaba, 2002. [Acesso em: 27/06/2016]. Disponível em: http://www. bibliotecadigital.unicamp.br/document/?c ode $=$ vtls000255945

15. Petersen KB. A hotel fire. Int Dent J. 1975; 25(3):172-78.

16. Kessler HP, Pemble CW. Forensic dental identification of casualties during Operation Desert Storm. Mil Med. 1993; 158(6): 359-62.

17. Raitz R, Fenyo-Pereira M, Hayashi AS, Melani R. Dento-maxillo-facial radiology as an aid to human identification. J. Forensic Odontostomatol. 2005; 23(2): 55-59. [Acesso em: 27/06/2016]. Disponível em: http://www.iofos.eu/ Jo urnals/JFOS\%20Dec05/Raitz.dec05.pdf

18. Morita MC, Haddad AE, Araújo ME. Perfil atual e tendências do cirurgiãodentista brasileiro. Maringá: Dental Press International. $96 \mathrm{p}$.

19. Nuto SAS, Noro LRA, Cavalsina PG, Costa ICC, Oliveira AGRC. O processo 
ensino-aprendizagem e suas consequiências na relação professor-alunopaciente. Ciênc Saúde Colet. 2006; 11(1):89-96. [Acesso em: 27/06/2016]. Disponível em: http://www.scielo. br/scielo.php?script=sci_arttext\&pid=S14 13-81232006000100016

20. Brasil. Ministério da Educação. Conselho Nacional de Educação. Câmara de Educação Superior. Resolução CNE/CES 3, de 19 fevereiro de 2002. Institui Diretrizes Curriculares Nacionais do Curso de Graduação em Odontologia. [Acesso em: 27/06/2016]. Disponível em: http://portal.mec.gov.br/cne/arquivos/pdf/
CES032002

21. Mendes EV, Marcos B. Odontologia integral: bases teóricas e suas implicações no ensino e na pesquisa odontológicas. Belo Horizonte: FINEP, 1985. 66p.

22. Paula LM, Bezerra ACB. A estrutura curricular dos cursos de Odontologia no Brasil. Rev ABENO. 2003;3(1):7-14.

Correspondência para:

Johnys Berton Medeiros da Nóbrega

e-mail: johnysberton@ gmail.com

Rua Antônio Vieira da Silva, 400/1101

Jardim São Paulo

58053-175 João Pessoa/PB 